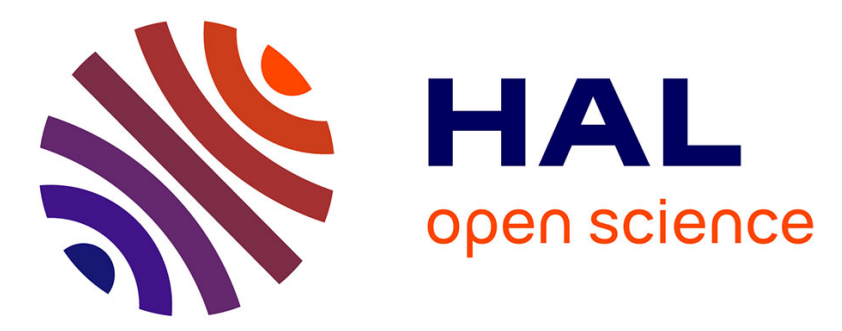

\title{
Experimental rheological procedure adapted to pasty dewatered sludge up to $45 \%$ dry matter
}

\author{
Mohamed Amine Mouzaoui, Jean-Christophe Baudez, Martial Sauceau, \\ Patricia Arlabosse
}

\section{- To cite this version:}

Mohamed Amine Mouzaoui, Jean-Christophe Baudez, Martial Sauceau, Patricia Arlabosse. Experimental rheological procedure adapted to pasty dewatered sludge up to $45 \%$ dry matter. Water Research, 2018, 133, pp.1-7. 10.1016/j.watres.2018.01.006 . hal-01678197

\section{HAL Id: hal-01678197 https://imt-mines-albi.hal.science/hal-01678197}

Submitted on 17 May 2018

HAL is a multi-disciplinary open access archive for the deposit and dissemination of scientific research documents, whether they are published or not. The documents may come from teaching and research institutions in France or abroad, or from public or private research centers.
L'archive ouverte pluridisciplinaire HAL, est destinée au dépôt et à la diffusion de documents scientifiques de niveau recherche, publiés ou non, émanant des établissements d'enseignement et de recherche français ou étrangers, des laboratoires publics ou privés. 


\title{
Experimental rheological procedure adapted to pasty dewatered sludge up to $45 \%$ dry matter
}

\author{
M. Mouzaoui a, b, J.C. Baudez ${ }^{\text {a, c, *, M. Sauceau }}{ }^{\text {b }}$, P. Arlabosse ${ }^{\text {b }}$ \\ a Irstea, UR TSCF, Domaine des Palaquins, F-03150 Montoldre, France \\ ${ }^{\mathrm{b}}$ Université de Toulouse, Mines Albi, CNRS, Centre RAPSODEE, Campus Jarlard, F-81013 Albi, France \\ ${ }^{\mathrm{c}}$ LIST Environmental Research and Innovation Department, 41 rue du Brill, L-4422 Belvaux, Luxembourg
}

Keywords:

Rheology

Pasty sludge

Restructuration

Viscoelasticity

Dilatancy

Fracture

\begin{abstract}
A B S T R A C T
Wastewater sludge are characterized by complex rheological properties, strongly dependent on solids concentration and temperature. These properties are required for process hydrodynamic modelling but their correct measurement is often challenging at high solids concentrations. This is especially true to model the hydrodynamic of dewatered sludge during drying process where solids content (TS) increases with residence time. Indeed, until now, the literature mostly focused on the rheological characterization of sludge at low and moderate TS (between 4 and 8\%). Limited attention was paid to pasty and highly concentrated sludge mainly because of the difficulties to carry out the measurements. Results reproducibility appeared to be poor and thus may not be always fully representative of the effective material properties. This work demonstrates that reproducible results can be obtained by controlling cracks and fractures which always take place in classical rotational rheometry. In that purpose, a well-controlled experimental procedure has been developed, allowing the exact determination of the surface effectively sheared. This surface is calculated by scattering a classical stress sweep with measurements at a reference strain value. The implementation of this procedure allows the correct determination of solidlike characteristics from 20 to 45\% TS but also shows that pasty and highly concentrated sludge highlight normal forces caused by dilatancy. Moreover the surface correction appears to be independent of TS in the studied range.
\end{abstract}

\section{Introduction}

Daily human activities generate a large quantity of wastewater. Before being discharged, these effluents have to be treated to minimize health risk and negative impact on the receiving environment. Treatments are carried out in wastewater treatment plant (WWTP) which inevitably generates a hazardous by-product to be eliminated, sludge. In the mid-2000s, the EU production was around 10 Mt of dry sludge (Fytili and Zabaniotou, 2008) and still increases with the combination of stricter regulation and population growth. Sludge management is a real challenge for municipalities around the world.

Because of landfilling banning and global opposition to land

\footnotetext{
* Corresponding author. LIST Environmental Research and Innovation Department, 41 rue du Brill, L-4422 Belvaux, Luxembourg.

E-mail addresses: mohamed_amine.mouzaoui@mines-albi.fr (M. Mouzaoui), jean-christophe.baudez@list.lu (J.C. Baudez).
}

application, the main route for sludge valorisation is currently energy recovery from incineration, pyrolysis or gasification. After the mechanical dewatering step total solid content (TS) is usually ranging from 20 to 30\% (Chen et al., 2002) but these TS contents are too low for energy valorisation. Indeed, $30-45 \%$ of TS content must be achieved for a specific incineration, $60-90 \%$ of solid content is required for co-incineration with municipal solid wastes, while more than $85 \%$ is expected for pyrolysis and gasification (Arlabosse et al., 2012; Ferrasse et al., 2002; Fytili and Zabaniotou, 2008). Thus thermal drying is required to reach these solid concentration levels (Arlabosse et al., 2012; Bennamoun, 2012; Bennamoun et al., 2013; Charlou et al., 2015; Chen et al., 2002; Ferrasse et al., 2002; Flaga, 2005).

However, thermal drying is highly energy-consuming (Arlabosse et al., 2012; Chabrier, 2007; Charlou et al., 2015; Kudra, 2003; Milhé et al., 2015) and, because of the difficulties to control the residence time in most agitated dryers, final TS content is often higher than what it is requested for the further thermal valorisation. Thus, process control to not overcome a given solid 
concentration threshold would save energy and money. This could be done by adjusting the residence time and so by controlling the flow rate which is intimately linked to sludge hydrodynamics and to sludge rheological properties.

Until now the literature mostly focused on the characterization of the rheological behavior of sludges at low (TS $<4 \%)$ and moderate $(4<\mathrm{TS}<17 \%)$ solid concentration (Baroutian et al., 2013; Baudez et al., 2013; Dong et al., 2011; Eshtiaghi et al., 2013; Farno et al., 2014; Jiang et al., 2014; Keiding et al., 2001; Lolito et al., 1997; Ma et al., 2014; Manoliadis and Bishop, 1984; Moeller and Torres, 1997; Mori et al., 2006; Pevere et al., 2009; Ratkovich et al., 2013; Ruiz-Espinoza et al., 2012; Ségalen et al., 2015; Wang et al., 2016, 2012). Limited attention was paid on pasty and highly concentrated sludge mainly because of the difficulties to carry out relevant measurements (Agoda-Tandjawa et al., 2013; Battistoni, 1997; Baudez et al., 2016; Baudez and Coussot, 2001; Chaari et al., 2003; Charlou, 2014; Jiang et al., 2014; Liang et al., 2017). Indeed, as the TS content increases, dominating interactions move from a lubricated regime (indirect contacts) to a frictional regime (direct contact), leading to dilatancy (Bagnold, 1966; Jaeger and Nagel, 1992; Phillips and Davies, 1991; Sosio et al., 2007; Van Damme et al., 2002) and fractures (Baudez and Coussot, 2001; Chaari et al., 2003; Coussot, 2014).

First, dilatancy is a common characteristic of granular pastes and concentrated colloidal suspensions. Discovered by Reynolds (1885) and detailed by Van Damme et al. (2002), dilatancy is described as follows: 'disordered dense packing of hard grains cannot undergo a shear without simultaneously expanding in the direction perpendicular to the shear plane'. In other words, the hard and compact particles are so close to each other that they have to push their neighbors to initiate their motion (Bagnold, 1966; Jaeger and Nagel, 1992), hence the interest of measuring the normal force simultaneously with tangential shear stress.

Second, edge effects taking the form of cracks and fractures occur when highly concentrated sludge is sheared. As a direct consequence, the effective sample contact area decreases (Ewoldt et al., 2015) leading to underestimated or unrealistic rheological properties: for example, no reliable steady-state data could be obtained with a highly concentrated tricalcium silicate paste due to fractures (Mansoutre et al., 1999). More generally, a comparison between cone partitioned plate (CPP) and parallel plates (PP) geometries on viscous polymers showed that results are only identical below a critical strain (Franck et al., 2012). Above this critical strain, CPP and PP geometries did not provide the same results because of fractures occurring with PP geometry. Fractures did not occur with CCP because such geometry allows a uniform shear (Cross and Kaye, 1986) but it can only be used with materials free of solids.

To avoid these perturbing effects (fractures), research done on highly concentrated sludge (Chaari et al., 2003) only focused on a low deformation range $($ strain $<30 \%$ ). For larger deformation, the experimental results were poorly reproducible because of the very likely occurrence of fracture. Thus, (large strain) flow curve determination of a highly concentrated sludge is still an unsolved challenge. But, as flow rates are usually low in dryers (Charlou, 2014; Ferrasse, 2000), shear rates are low as well and solid-like properties can be considered as dominant in this range of velocities (Baudez et al., 2011). In consequence, the evolution of rheological properties during drying could be reasonably assumed to be similar

Table 1

Measurement of TS and VS content after centrifugation.

\begin{tabular}{lllll}
\hline Centrifugation time (h) & 0 & 0.5 & 1.5 & 24 \\
\hline TS content (wt. \%) & 20.6 & 29.7 & 34.3 & 44.2 \\
VS content (wt. \% TS) & 63.3 & 63.4 & 63.6 & 63.3 \\
\hline
\end{tabular}

to solid-like properties which can be accurately determined with adapted precautions.

In this work, a specific procedure has been developed to systematically take into account edge effects and to allow the investigation of viscoelastic solid-like properties of pasty sludge by using a simple geometry, compatible with any commercial rotational rheometer.

\section{Material and methods}

\subsection{Material}

Dewatered anaerobically digested sludge was sampled at the outlet of the centrifuge step at the WWTP from Albi city (France). Its initial TS content was $20 \%$ and samples of different TS content ranging from 8 to $45 \%$ have been prepared by lab centrifugation (at $30000 \mathrm{rpm}$ or $108800 \mathrm{~g}$ ) for duration from $30 \mathrm{~min}$ to $24 \mathrm{~h}$ at room temperature or by dilution (with addition of deionised water).

Volatile solid content of total solids (VS) has been determined for several samples to check that centrifuge did not alter sludge composition (Table 1 ).

Sludge was then stored at $4{ }^{\circ} \mathrm{C}$ for at least $24 \mathrm{~h}$ to ensure full homogenization.

Biological stability over time of samples was checked after sampling. It was found that sludge can be considered biologically stable for a period of 10 days as rheological characteristics did not evolve during this period of time (Fig. 1). Thus, samples were only considered for 10 days, to ensure reproducible measurements.

\subsection{Rheological measurements}

Rheological measurements were performed with stresscontrolled RS600 instrument (HAAKE) piloted by RheoWin software at ambient temperature. Serrated (to avoid wall slip) parallel plate geometries (radius 10 and $17.5 \mathrm{~mm}$ ) were used with a gap set at $2 \mathrm{~mm}$. Only dynamic mode was used in this work.

For parallel plates, shear stress and shear strain are calculated from the geometric parameters (plates radius and gap) and raw measurements (torque and rotational angle) as follows (HAAKE, 1999):

$$
\begin{aligned}
& \tau_{\mathrm{i}}=\frac{2 \cdot \mathrm{M}_{\mathrm{i}}}{\pi \cdot \mathrm{R}(0)^{3}} \\
& \gamma_{\mathrm{i}}=\frac{\varphi_{\mathrm{i}} \cdot \mathrm{R}(0)}{\mathrm{h}}
\end{aligned}
$$

Where $\tau_{\mathrm{i}}, \gamma_{\mathrm{i}}, \mathrm{M}_{\mathrm{i}}, \varphi_{\mathrm{i}}, \mathrm{R}(0)$ and $\mathrm{h}$ represent the shear stress [Pa], the

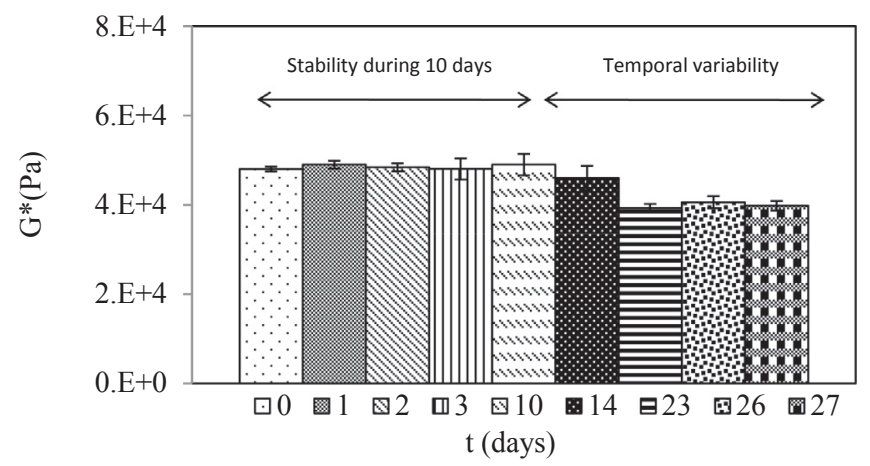

Fig. 1. Biological stability of digested sludge at $\mathrm{TS}=20 \%$. The rheological properties remain almost constant for 10 days after sampling. 


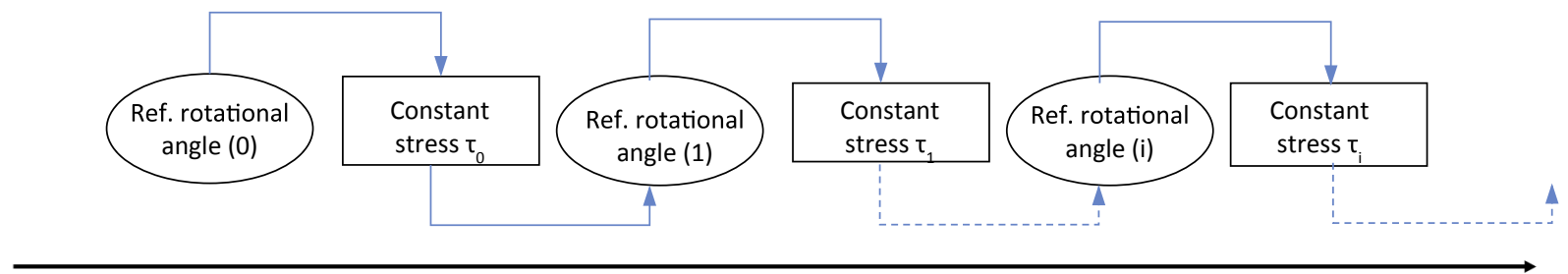

Experimental time

Fig. 2. Experimental procedure allowing to recalculate the effective really sheared radius.

shear strain [\%], the torque [N.m], the rotational angle [rad], the radius of the plates $[\mathrm{m}]$ and the gap between the plates $[\mathrm{m}]$, respectively. Stress and strain ratio allows the calculation of the complex modulus, $G_{i}^{*}$ :

$\mathrm{G}_{\mathrm{i}}^{*}=\frac{\tau_{\mathrm{i}}}{\gamma_{\mathrm{i}}}=\frac{2 \cdot \mathrm{h} \cdot \mathrm{M}_{\mathrm{i}}}{\pi \cdot \varphi_{\mathrm{i}} \cdot \mathrm{R}(0)^{4}}$

The loss (or viscous) modulus $G_{i}^{\prime \prime}$ and the storage (or elastic) modulus $G_{i}^{\prime}$ are then derived from $G_{i}^{*}$. $G_{i}^{\prime \prime}$ represents the viscous dissipation or loss of energy, while $G_{i}^{\prime}$ characterizes the energy stored or how well structured the material is.

For different solid concentrations, a stress sweep consisting in successive steps of constant dynamic stress $\tau_{i}$ (corresponding to a torque $\mathrm{M}_{\mathrm{i}}$ ) of increasing intensity (10 points per decade) has been applied to the sample. A pause is marked between each step. Only one constant dynamic stress at $1 \mathrm{~Hz}$ is applied by step. Each constant dynamic stress was preceded (during the pause) by a reference test allowing the correction of edge effects (Fig. 2). Despite the stress-controlled mode, it has been decided to carry out this reference test by applying a constant dynamic rotational angle $\varphi_{\text {ref }}$ in the linear viscoelastic range (LVE), where, by definition, stress and strain are proportional (the elastic modulus being constant). But, because edge effects induce a decrease of the effective shear surface, applying a constant reference torque would have driven to an uncontrolled increase of the stress and so of the strain, potentially out of the LVE range. So, by applying this constant dynamic rotational angle, edge effects drive to a decrease of the corresponding stress ensuring to remain in the LVE range. An initial dynamic rotational angle of $0.6 \mathrm{mrad}$ (corresponding to a reference strain $\gamma_{\text {ref }}$ of $0.3 \%$ without edge effects) has been found to be in the LVE range with the $10 \mathrm{~mm}$ plate. Only one constant dynamic rotational angle $(0.6 \mathrm{mrad})$ at $1 \mathrm{~Hz}$ is applied by reference test.

This reference rotational angle $\varphi_{\text {ref }}$ has also been considered to evaluate the restructuration kinetics of sludge by applying a constant dynamic strain over time and measuring the evolution of rheological characteristics ( 1 measurement every $10 \mathrm{~s}$ ). Because a strain of $0.3 \%$ is found to be in the LVE range, the number of measurement applied does not matter. This step will help at defining the appropriate time of rest needed to neglect restructuration effects during the experimental procedure which allows recalculating the effective really sheared radius.

Tests have been made in triplicate to evaluate the reproducibility.

Normal force was recorded in parallel with shear characteristics.

\section{Results and discussion}

\subsection{Restructuration}

When submitted to a constant strain in the linear viscoelastic regime, the loss tangent slowly but continuously decreases (Fig. 3), indicating a restructuration is occurring and the material is becoming more and more solid (Baudez, 2008).

To ensure restructuration effects will not impact results during measurements (stress sweep measurements scattered with the reference rotational angle), it has been decided to apply a preliminary constant dynamic strain plateau at $0.3 \%$ (the reference strain $\gamma_{\text {ref }}$ ) for $300 \mathrm{~s}$. Beyond this time, restructuration only evolved by $2 \%$ in $20 \mathrm{~min}$, which is considered as negligible regarding the signal intensity.

\subsection{Stress sweep measurements}

As the stress increases, a fracture appears on the edges above a critical strain and progressively spreads to the whole volume (Fig. 4). Because rheological characteristics are calculated by the rheometer software from raw torque and rotational angle on the basis of a full sheared surface, the impact of this fracture has to be corrected.

This correction implies to exactly know for each level of stress (or torque) the real surface which is effectively sheared (Fig. 5). This can be done by considering the complex modulus $\mathrm{G}^{*}$ in the LVE range, which is by definition constant, assuming the innerstructure of sludge has not been impacted by the applied shear. To ensure this assumption remains valid during our experiments, the largest strain (to be corrected) has been limited to $200 \%$, corresponding to a rotational angle of $400 \mathrm{mrad}$ with the $10 \mathrm{~mm}$ plate.

Thus, the effective radius can be recalculated with the following procedure. First, the reference strain $\gamma_{\text {ref }}$ is given by equation (4):

$\gamma_{\text {ref }}=\frac{\varphi_{\text {ref }} \cdot \mathrm{R}(0)}{\mathrm{h}}$

$R(0)$ is the initial radius of sample ( $m)$, which is equal to the plate one, and $\varphi_{\text {ref }}$ is the constant reference rotational angle $(0.6 \mathrm{mrad})$.

Taking into account progressive edge effects, applying $\varphi_{\text {ref }}$

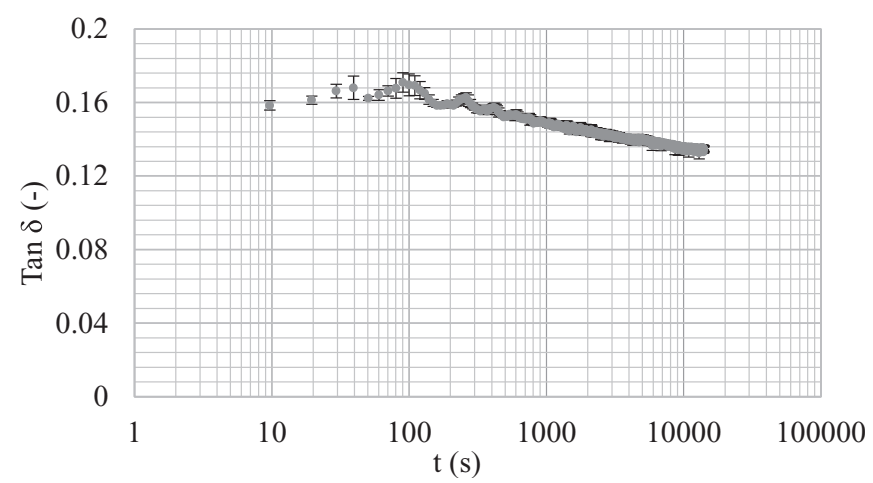

Fig. 3. Evolution of loss tangent as function of time under a constant strain for sludge at TS $=20 \%$. 

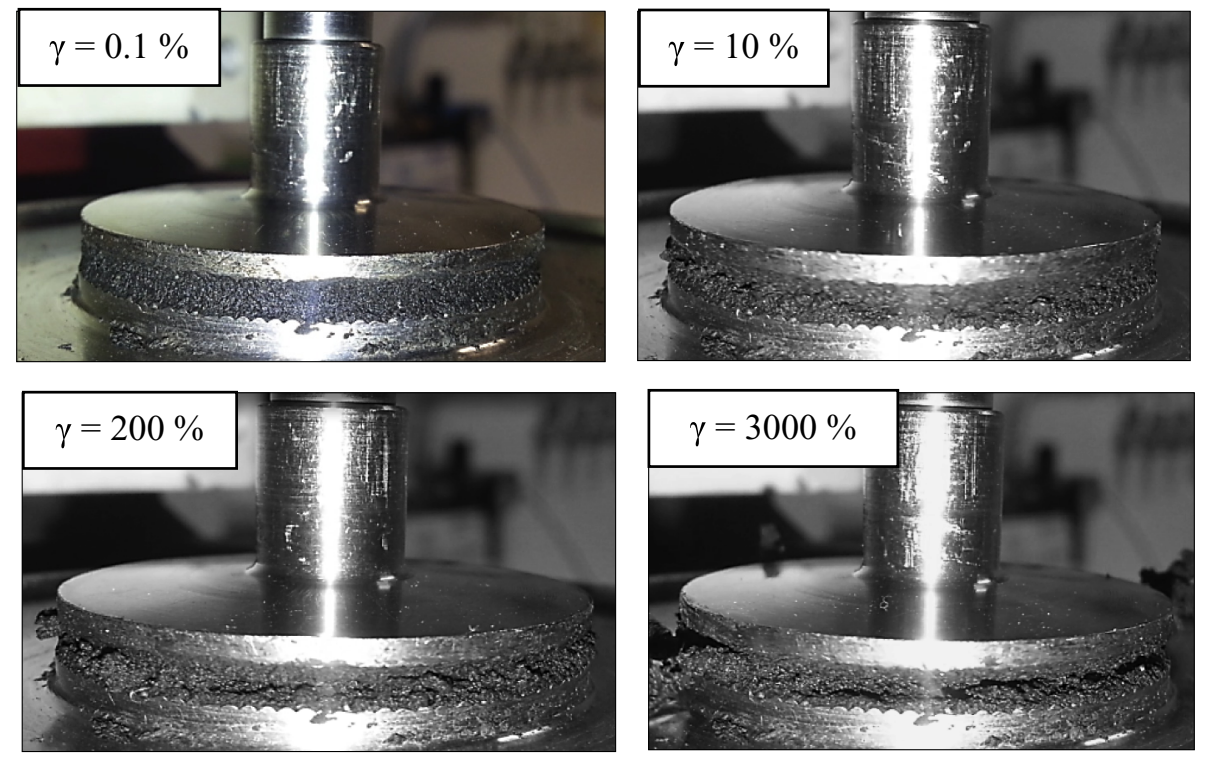

Fig. 4. Visualization of the edge fracture sheared between parallel plates during stress sweep experiment (at TS=20\%).

drives to a shear stress equal to:

$\tau_{\text {ref }}(\mathrm{i})=\frac{2 \cdot \mathrm{M}_{\mathrm{ref}}(\mathrm{i})}{\pi \cdot \mathrm{R}(\mathrm{i})^{3}}$

At the beginning, before the fracture has appeared, the measured torque is equal to $\mathrm{M}_{\mathrm{ref}}(0)$ and the complex modulus $\mathrm{G}_{\mathrm{ref}}^{*}(0)$ in this reference state (corresponding to $\mathrm{R}(0)$ ) is thus:

$\mathrm{G}_{\mathrm{ref}}^{*}(0)=\frac{\tau_{\mathrm{ref}}(0)}{\gamma_{\mathrm{ref}}}=\frac{2 \cdot \mathrm{h} \cdot \mathrm{M}_{\mathrm{ref}}(0)}{\pi \cdot \varphi_{\mathrm{ref}} \cdot \mathrm{R}(0)^{4}}$

Where $R(i)$ corresponds to the effectively sheared radius and $\mathrm{M}_{\text {ref }}(\mathrm{i})$ the effective torque when the reference rotational angle is applied.

Complex modulus at the reference rotational angle is then defined by the following equation:

$\mathrm{G}_{\text {ref }}^{*}(\mathrm{i})=\frac{\tau_{\text {ref }}(\mathrm{i})}{\gamma_{\text {ref }}}=\frac{2 \cdot \mathrm{h} \cdot \mathrm{M}_{\mathrm{ref}}(\mathrm{i})}{\pi \cdot \varphi_{\text {ref }} \cdot \mathrm{R}(\mathrm{i})^{4}}$

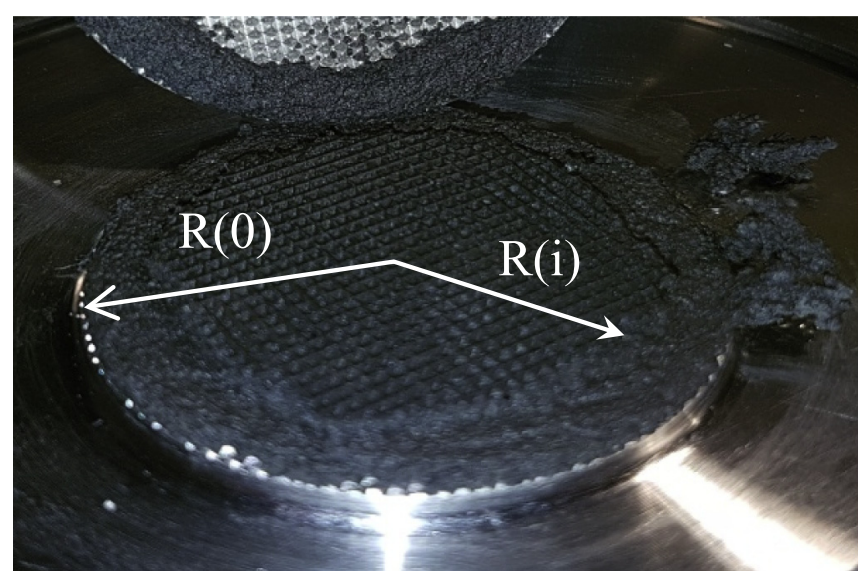

Fig. 5. Picture of the surface really sheared $\left(S=\pi \cdot R(i)^{2}\right)$ between parallel plates at the end of the stress sweep ( $3000 \%$ of strain). $R(0)$ is the initial radius.
Assuming the inner-sludge structure is not impacted at these low levels of deformation, the complex modulus can be considered constant at the reference state before and after the fracture. Then it comes:

$\mathrm{G}_{\text {ref }}^{*}(\mathrm{i})=\mathrm{G}_{\text {ref }}^{*}(0)$

Where $\mathrm{G}_{\mathrm{ref}}^{*}(0)$ represents the initial (before the fracture) complex modulus at $\mathrm{R}(\mathrm{i})=\mathrm{R}(0)$.

Combining Equation (7) and Equation (8), it comes:

$\frac{\mathrm{M}_{\mathrm{ref}}(\mathrm{i})}{\mathrm{R}(\mathrm{i})^{4}}=\frac{\mathrm{M}_{\mathrm{ref}}(0)}{\mathrm{R}(0)^{4}}$

And so:

$\mathrm{R}(\mathrm{i})=\mathrm{R}(0) \cdot \sqrt[4]{\frac{\mathrm{M}_{\mathrm{ref}}(\mathrm{i})}{\mathrm{M}_{\mathrm{ref}}(0)}}$

Then the radius really sheared obtained from each reference state allows correcting the stress (and the corresponding strain) as shown on Fig. 6.

The Table 2 shows an example of raw data obtained with a $10 \mathrm{~mm}$ plate. Using equation (10), the radius really sheared can be calculated at the reference state (i) as follows:

$\mathrm{R}(\mathrm{i})=10 \cdot \sqrt[4]{\frac{217}{285}}=9.34 \mathrm{~mm}$

Then, stress and strain can be corrected using this radius $\mathrm{R}(\mathrm{i})$.

$\tau_{\mathrm{i}}=\frac{2 \cdot \mathrm{M}_{\mathrm{i}}}{\pi \cdot \mathrm{R}(\mathrm{i})^{3}}=\frac{2 \cdot 9468 \cdot 10^{-6}}{\pi \cdot\left(9.34 \cdot 10^{-3}\right)^{3}}=7400 \mathrm{~Pa}$

$\gamma_{\mathrm{i}}=\frac{\varphi_{\mathrm{i}} \cdot \mathrm{R}(\mathrm{i})}{\mathrm{h}}=\frac{154 \cdot 10^{-3} \cdot 9.34}{2}=72 \%$

Finally, these intrinsic stress and strain allow calculating the corrected complex modulus: 


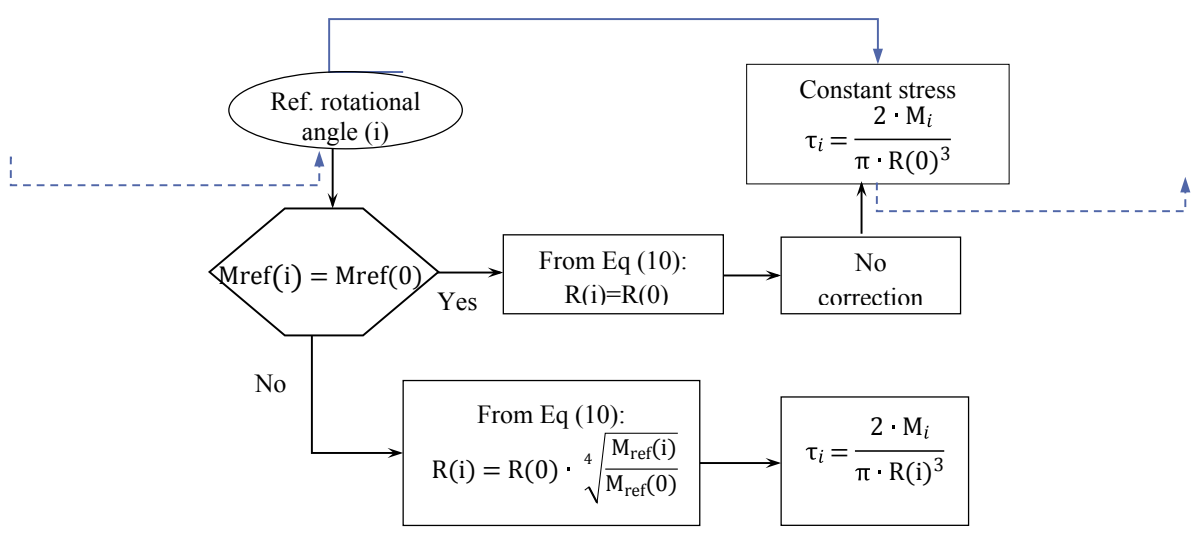

Fig. 6. How the corrected radius from each reference rotational state is used to correct the rheological parameters.

Table 2

Example of raw data at some reference states. Initial radius is $\mathrm{R}(0)=10 \mathrm{~mm}$.

\begin{tabular}{llll}
\hline Applied measurements & $\mathrm{M}(\mu \mathrm{Nm})$ & $\varphi(\mathrm{mrad})$ & $\delta\left(^{\circ}\right)$ \\
\hline Initial Ref. rotational angle $(0)$ & 285 & 0.6 & 8.5 \\
Ref. rotational angle (i-1) & 249 & 0.6 & 8.5 \\
Constant stress $\tau_{\mathrm{i}-1}$ & 6297 & 42 & 18.3 \\
Ref. rotational angle $(\mathrm{i})$ & 217 & 0.6 & 8.5 \\
Constant stress $\tau_{\mathrm{i}}$ & 9468 & 154 & 31.0 \\
\hline
\end{tabular}

$\mathrm{G}_{\mathrm{i}}^{*}=\frac{\tau_{\mathrm{i}}}{\gamma_{\mathrm{i}}}=\frac{7400}{0.72}=10280 \mathrm{~Pa}$

Then, from the corrected complex modulus $\mathrm{G}^{*}$ and the loss angle $\delta$ (Table 2), which is not impacted by the surface correction, the effective value of the loss modulus $G^{\prime \prime}$ and the storage modulus $G^{\prime}$ can be determined as follow (Malvern Instruments, 2016):

$\mathrm{G}^{\prime}=\mathrm{G}_{\mathrm{i}}^{*} \cdot \cos \delta_{i}=10280 \cdot \cos \left(31^{\circ}\right)=8840 \mathrm{~Pa}$

$\mathrm{G}^{\prime \prime}=\mathrm{G}_{\mathrm{i}}^{*} \cdot \sin \delta_{i}=10280 \cdot \sin \left(31^{\circ}\right)=5300 \mathrm{~Pa}$

The evolution of the surface really sheared $S(i)=\pi \cdot R(i)^{2}$ as function of strain during measurements evidenced two regions separated by a critical strain $\gamma_{c}$ (Fig. 7) above which the surface really sheared is no more equal to the initial one indicating that fractures occur. It first starts to decrease slightly as the strain increases, then, a strong decrease is observed.

Evolutions of elastic and viscous moduli as a function of the

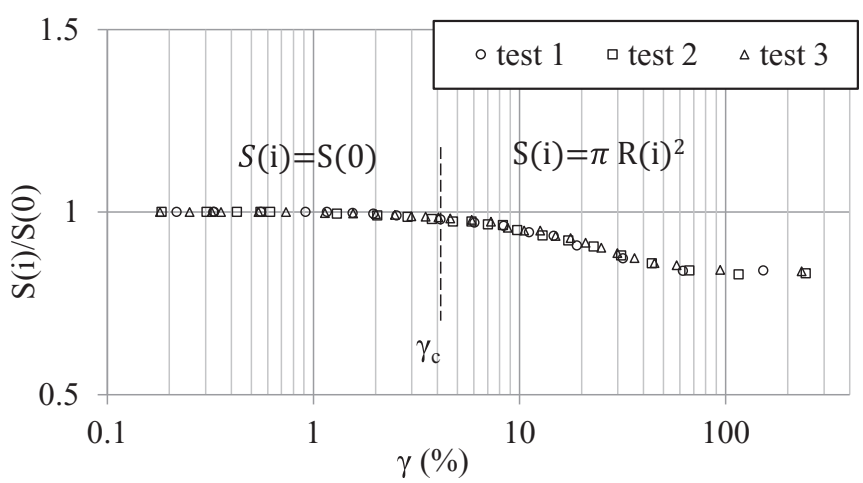

Fig. 7. Evolution of the surface ratio during obtained by the experimental procedure for the sludge at TS $=20 \%$. $\left(S(0)=\pi \cdot R(0)^{2}\right.$ is the initial surface).

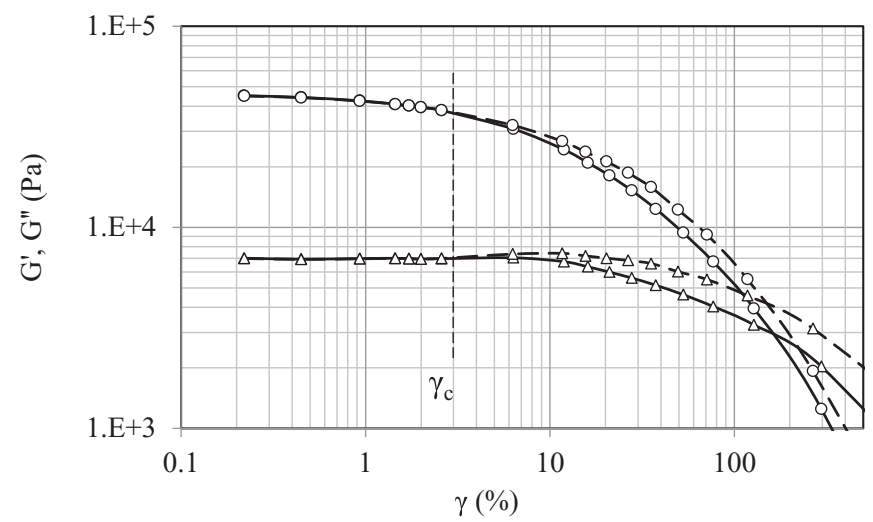

Fig. 8. Evolution of storage $(O)$ and loss $(\Delta)$ moduli during rheological measurements with (- - ) and without (-) surface correction for the sludge at TS $=20 \%$.

strain with and without the surface correction are displayed in Fig. 8. The global shape of $G^{\prime}$ and $G^{\prime \prime}$ does not change but the corrected values differ from the uncorrected ones up to $40 \%$ (Table 3 and Fig. 8).

Corrected data showed that $\mathrm{G}^{\prime}$ starts to become straindependent above $0.5 \%$ before sharply decreasing above $10-15 \%$ while $G^{\prime \prime}$ remains almost strain-independent in this range. Then, at higher strain, moduli decrease together until a crossover point at around $160 \%$.

Normal force measurement and corrected surface allow the calculation of the normal stress defined by $\sigma=\mathrm{Fn} / \mathrm{S}[\mathrm{Pa}]$ which clearly highlights specific regions (Fig. 9) with 3 distinct zones: a decrease below the critical strain $\gamma_{c}$, then a sudden increase before an abrupt fall at $\gamma_{f}$ corresponding to the decrease of G". The sudden jump is typical of dilatancy in granular media (Mansoutre et al., 1999).

The onset of dilatancy corresponds closely to the critical strain. Indeed, particles cannot move independently from each other and

Table 3

Evolution between corrected and uncorrected data (corrected by uncorrected) at different strains.

\begin{tabular}{llll}
\hline$\gamma(\%)$ & $\mathrm{S}(\mathrm{i}) / \mathrm{S}(0)$ & $\tau_{\mathrm{i}} / \tau_{\mathrm{i}}$ & $\mathrm{G}_{\mathrm{i}}^{*} / \mathrm{G}_{\mathrm{i}}^{*}$ \\
\hline 3 & 0.99 & 1.01 & 1.02 \\
15 & 0.94 & 1.10 & 1.13 \\
150 & 0.84 & 1.30 & 1.42 \\
\hline
\end{tabular}




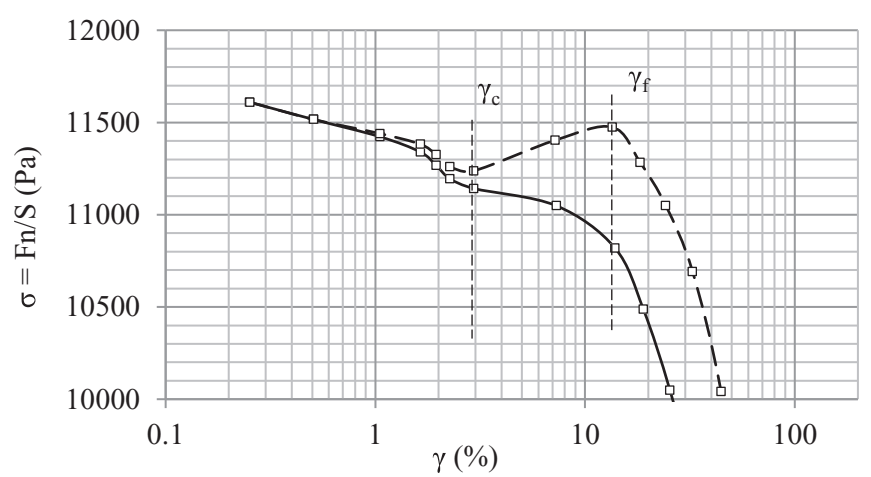

Fig. 9. Evolution of the normal stress during rheological measurements with (- - -) and without (-) surface correction for the sludge at TS $=20 \%$.

they need to push their neighbors. It results in a movement in the direction perpendicular to the shear plane (Van Damme et al., 2002) leading to an increase of the normal stress.

Then, normal stress abruptly decreases in parallel with $G^{\prime \prime}$, above $\gamma_{\mathrm{f}}$. Thus it can be assumed that the decrease of the normal stress results from viscous-like dissipation which takes place in the material: the structure dissipates its internal energy through fractures.

Finally, at higher strain, moduli decrease together until a crossover point at a strain around $160 \%$, marking the transition from a viscoelastic solid characteristic to a viscoelastic liquid property (Jiang et al., 2014). Beyond the crossover point, $\mathrm{G}^{\prime \prime}$ becomes higher than $G^{\prime}$ as the strain increases, reflecting the now essentially viscous nature of the medium as noticed by Mansoutre et al. (1999).

\subsection{TS content impact on surface correction}

Fracture intensity depends on TS (Fig. 10). As long as TS is low ( $14 \%$ in our case), when measuring tools are moved away, the surface profile looks like a cone reflecting that the surface was fully sheared (no fracture). Then, for TS higher than $14 \%$, the surface profile displays concentric circles and a plane surface in the centre, reflecting the existence of fracture.

Interestingly, as soon as fracture is occurring, whatever the TS (ranging from 14 to $45 \%$ ), the surface correction curves are superimposed (Fig. 11), indicating the same phenomena - but probably at different intensities - occurs (Baudez et al., 2011).

The existence of a single curve for the surface-correction means that, in this range of TS, a single surface-correction curve could be sufficient to obtain intrinsic characteristics for any other TS.

This procedure allows to accurately determine the rheological viscoelastic solid-like characteristics of highly dewatered sludge. The door is thus open to define how these (correctly measured) parameters evolve with solid concentration and temperature as it is the case in dryers.

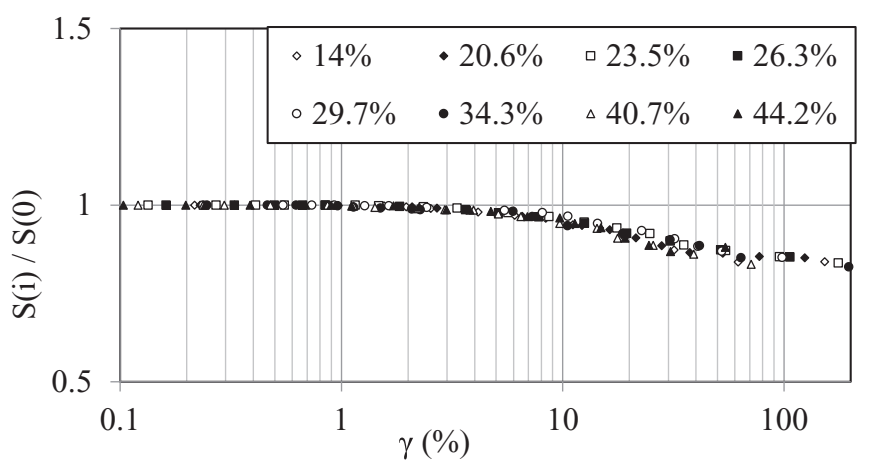

Fig. 11. -The evolution of the surface ratio during the experimental procedure of correction of sludge is similar for all the tested concentrations $(14<\mathrm{TS}<44 \%)(\mathrm{S}(0)$ : the initial surface).

The evolution of these parameters will allow the definition of a kinetic function regarding temperature and concentration changes during drying process. Thus, knowing the rheological properties of sludge at the inlet and its kinetics, the residence time can be evaluated and adapted to control the final solid concentration which will correspond, for a single sludge, to a given elastic modulus.

\section{Conclusion}

Literature has shown that rheological measurements of pasty sludge are limited to low strains and may require complex configurations (which are not accessible for any commercial rheometer) because of uncontrolled effects such as cracks and fractures.

This work demonstrates how cracks and fractures in rotational rheometry can be successfully controlled in order to obtain intrinsic rheological parameters, using a simple geometry, compatible with any commercial rotational rheometer. The experimental procedure is based on a sheared surface correction. This correction is determined in real time by scattering a classical stress sweep with measurements at a reference strain value. It is also shown that the surface correction is independent of the total solids content (TS) in the studied range (14-45\%), resulting in a single correction curve. Moreover, the implementation of this procedure showed that pasty and highly concentrated sludges present similarities with granular material (dilatancy or jamming).

The overall results of this research pave the road of a more complete modelling of the hydrodynamic behavior of sludge during drying processes. Indeed, the knowledge of the sludge rheological properties on a wide range of TS allows a better understanding of the flow evolution all along the dryer. This will drive to a better control of the residence time and thus the final solid content.

Future works will be however needed to better understand and potentially to model the transition from lubrificated to frictional regimes, and how the frictional contribution can impact the rheological parameters.
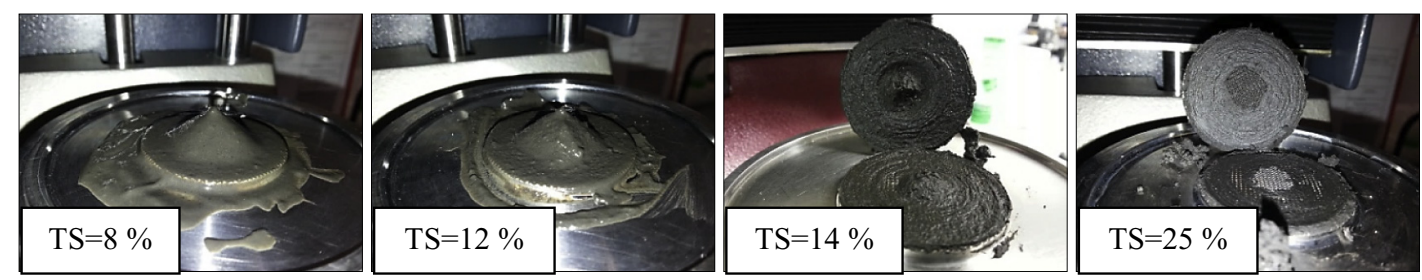

Fig. 10. Surface profile of sludge between parallel plates at the end of experiments 


\section{References}

Agoda-Tandjawa, G., Dieudé-Fauvel, E., Girault, R., Baudez, J.C., 2013. Using water activity Chemical, measurements to evaluate rheological consistency and structure strength of sludge. Eng. J. 228 (0), 799-805.

Arlabosse, P., Ferrasse, J.H., Lecompte, D., Crine, M., Dumont, Y., Léonard, A., 2012. Efficient sludge thermal processing: from drying to thermal valorisation. In Modern Drying Technology: Energy Savings 4, 295-329.

Bagnold, R., 1966. The shearing and dilatation of dry sand and the "singing" mechanism. Proc Roy Soc A 295, 219-232.

Baroutian, S., Eshtiaghi, N., Gapes, D., 2013. Rheology of a primary and secondary sewage sludge mixture: dependency on temperature and solid concentration. Bioresour. Technol. 140, 227-233.

Battistoni, P., 1997. Pre-treatment, measurement execution procedure and waste characteristics in the rheology of sewage sludges and the digested organic fraction of municipal solid wastes. Water Sci. Technol. 3611, 33-41.

Baudez, J.C., 2008. Physical aging and thixotropy in sludge rheology. Appl. Rheol. 18, 13459-13466.

Baudez, J.C., Coussot, P., 2001. Rheology of aging, concentrated, polymeric suspensions e Application to pasty sewage sludges. J. Rheol. 45 (5), 1123-1139.

Baudez, J.C., Gupta, R.K., Eshtiaghi, N., Slatter, P., 2013. The viscoelastic behaviour of raw and anaerobic digested sludge: strong similarities with soft-glassy materials. Water Res. 47, 173-180.

Baudez, J.C., Markis, F., Eshtiaghi, N., Slatter, P., 2011. The rheological behaviour of anaerobic digested sludge. Water Res. 45, 5675-5680.

Baudez, J.C., Megnien, J.C., Guibelin, E., 2016. Pumping of dewatered Sludge : slipping or flowing behavior? Chem. Eng. J. 295, 494-499.

Bennamoun, L., 2012. Solar drying of wastewater sludge : a review. Renew. Sustain. Energy Rev. 16 (1), 1061-1073.

Bennamoun, L., Arlabosse, P., Léonard, A., 2013. Review on fundamental aspect of application of drying process to wastewater sludge. Renew. Sustain. Energy Rev. $28,29-43$.

Chaari, F., Racineux, G., Poitou, A., Chaouche, M., 2003. Rheological behavior of sewage sludge and strain-induced dewatering. Rheol. Acta 42, 273-279.

Chabrier, J.P., 2007. Le séchage thermique des boues: le développement, ses avantages et ses inconvénients. In: Journée Technique Du SIAAP.

Charlou, C., 2014. Caractérisation et modélisation de l'écoulement de boues résiduaires dans un sécheur à palettes. In: Rapsodee, Ecole des Mines d'AlbiCarmaux.

Charlou, C., Milh, M., Sauceau, M., Arlabosse, P., 2015. A new methodology for measurement of sludge residence time distribution in a paddle dryer using Xray fluorescence analysis. Water Res. 69, 1-8.

Chen, G.H., Yue, P.L., Mujumdar, A.S., 2002. Sludge dewatering and drying. Technol. Drying 20, 883-916.

Coussot, P., 2014. Yield stress fluid flows: a review of experimental data. J. NonNewtonian Fluid Mech. 211, 31-49.

Cross, M., Kaye, A., 1986. Techniques for the viscometry of suspensions. Polym. Eng. Sci. 26, 121-126.

Dong, Y.J., Wang, Y.L., Feng, J., 2011. Rheological and fractal characteristics of unconditioned and conditioned water treatment residuals. Water Res. 45, 3871-3882.

Eshtiaghi, N., Markis, F., Yap, S.D., Baudez, J.C., Slatter, P., 2013. Rheological characterisation of municipal sludge: a review. Water Res. 47, 5493-5510.

Ewoldt, R.H., Johnston, M.T., Caretta, L.M., 2015. Experimental Challenges of Shear Rheology: How to Avoid Bad Data, pp. 1-36.

Farno, E., Baudez, J.C., Parthasarathy, R., Eshtiaghi, N., 2014. Rheological characterisation of thermally-treated anaerobic digested sludge: impact of temperature and thermal history. Water Res. 56, 156-161.

Ferrasse, J.H., 2000. Développement d'outils expérimenataux pour le dimensionnement du procédé de séchage conductif avec agitation : application à des boues de station d'épuration urbaines. Thèse Univ. Toulouse II.

Ferrasse, J.H., Arlabosse, P., Lecomte, D., 2002. Heat, momentum, and mass transfer measurements in indirect agitated sludge dryer. Dry. Technol. 20 (4-5), 749-769.
Flaga, A., 2005. Sludge drying, proceedings of Polish-Seminars. Cracow 73-82.

Franck, A., Klein, C.M.W., 2012. Partitioned plate/cone to perform large amplitude oscillation shear (Laos) measurements on highly viscous fluids (POSTER). In: XVIth Congress on Rheology Held. August 5-10, 2012 at Lisbon, Portugal. Lisbon.

Fytili, D., Zabaniotou, A., 2008. Utilization of sewage sludge in EU application of old and new methods: a review. Renew. Sustain. Energy Rev 12, 116-140.

HAAKE, 1999. Instruciton manual for the measuring instruments. In: Instruction Manual Rheostress RS75/RS80 and RS150.

Jaeger, H., Nagel, S., 1992. Physics of the granular state. Science 255, 1523-1531.

Jiang, J., Wu, J., Poncin, S., Li, H.Z., 2014. Rheological characteristics of highly concentrated anaerobic digested sludge. Biochem. Eng. J. 86, 57-61.

Keiding, K., Wybrandt, L., Nielsen, P.H., 2001. Remember the water: comment on EPS colligative properties. Water Sci. Technol. 43, 17-23.

Kudra, T., 2003. Sticky region in drying-definition and identification. Dry. Technol. 21 (8), 1457-1469.

Liang, F., Sauceau, M., Dusserre, G., Arlabosse, P., 2017. A uniaxial cyclic compression method for characterizing the rheological and textural behaviors of mechanically dewatered sewage sludge. Water Res. 113, 171-180.

Lolito, V., Spinosa, L., Mininni, G., Antonacci, R., 1997. The rheology of sewage sludge at different steps of treatment. Water Sci. Technol. 36 (11), 79-85.

Ma, Y., Xia, C., Yang, H., Zeng, R.J., 2014. A rheological approach to analyze aerobic granular sludge. Water Res. 50, 171-178.

Malvern Instruments, 2016. A Basic Introduction to Rheology.

Manoliadis, O., Bishop, P., 1984. Temperature effect on rheology of sludges. J. Environ. Eng. 1, 286-290.

Mansoutre, S., Colombet, P., Van Damme, H., 1999. Water retention and granular rheological behavior of fresh C3S paste as a function of concentration. Cement Concr. Res. 29, 1441-1453.

Milhé, M., Charlou, C., Sauceau, M., Arlabosse, P., 2015. Modeling of sewage sludge flow in a continuous paddle dryer. Dry. Technol. 33, 1061-1067.

Moeller, G., Torres, L.G., 1997. Rheological characterization of primary and secondary sludges treated by both aerobic and anaerobic digestion. Bioresour. Technol. 61 (8), 207-211.

Mori, M., Seyssiecq, I., Roche, N., 2006. Rheological measurements of sewage sludge for various solids concentrations and geometry. Process Biochem. 41, 1656-1662.

Pevere, A., Guibaud, G., Goin, E., van Hullebusch, E., Lens, P., 2009. Effects of physicochemical factors on the viscosity evolution of anaerobic granular sludge. Biochem. Eng. J. 43, 231-238.

Phillips, C.J., Davies, T.R.H., 1991. Determining rheological properties of debris flow material. Geomorphology 4, 101-110.

Ratkovich, N., Horn, W., Helmus, F.P., Rosenberger, S., Naessens, W., Nopens, I., Bentzen, T.R., 2013. Activated sludge rheology: a critical review on data collection and modelling. Water Res. 47, 463-482.

Reynolds, O., 1885. On the dilatancy of media composed of rigid particles in contact. With experimental illustrations. Phil. Mag. 20, 469.

Ruiz-Espinoza, J.E., Méndez-Contreras, J.M., Alvarado-Lassman, A., MartínezDelgadillo, S.A., 2012. Effect of low temperature thermal pre-treatment on the solubilization of organic matter, pathogen inactivation and mesophilic anaerobic digestion of poultry sludge. J. Environ. Sci. Health: Part A 47, 1795-1802.

Ségalen, C., Dieudé-Fauvel, E., Clément, J., Baudez, J.C., 2015. Relationship between electrical and rheological properties of sewage sludge - impact of temperature. Water Res. 73, 1-8.

Sosio, R., Crosta, B.G., Frattini, P., 2007. Field observations, rheological testing and numerical modeling of a debris-flow event. Earth Surf. Process. Landforms 32, 290-306.

Van Damme, H., Mansoutre, S., Colombet, P., Lesaffre, C., Picart, D., 2002. Pastes: lubricated and cohesive granular media. Compt. Rendus Phys. 3, 229-238.

Wang, H., Hu, H., Yang, H., Zeng, R., 2016. Characterization of anaerobic granular sludge using a rheological approach. Water Res. 106, 116-125.

Wang, Y., Dong, Y., Feng, J., 2012. Scaling behaviors of unconditioned and conditioned water treatment residuals (WTRs) based on rheological and microscopic characterization. Colloid. Surface. Physicochem. Eng. Aspect. 402, 152-158. 